\title{
Palinología de las especies mexicanas de Gleicheniaceae
}

\author{
Norberto Farfán-Santillán ${ }^{1}$, Aniceto Mendoza-Ruiz ${ }^{1}{ }^{3}$, Blanca Pérez- \\ García $^{1}$ y ERnesto VelázQuez-Montes ${ }^{2}$
}

Botanical Sciences

94 (2): 281-289, 2016

DOI: $10.17129 /$ botsci.509

\section{Resumen}

Se realizó el estudio palinológico de las esporas de Dicranopteris flexuosa, Diplopterygium bancroftii, Gleichenella pectinata, Sticherus bifidus, S. brevipubis, S. palmatus y S. underwoodianus, especies mexicanas de la familia Gleicheniaceae. El material fue recolectado en diversas localidades del país, las descripciones morfológicas se realizaron con base en observaciones hechas con microscopía óptica y electrónica de barrido, se incluyen imágenes para cada una de las especies. Los resultados muestran que se presentan diferencias entre las especies en el tipo de lesura y ornamentación. Se describe por primera vez la micromorfología de las esporas de $S$. brevipubis, S. palmatus y $S$. underwoodianus.

Palabras clave: esporas, microscopía electrónica de barrido, ornamentación, Pteridophyta.

\section{Palinology of the mexican species of Gleicheniaceae Abstract}

The palynological study of the spores of Dicranopteris flexuosa, Diplopterygium bancroftii, Gleichenella pectinata, Sticherus bifidus, S. brevipubis, S. palmatus, and S. underwoodianus, which are all Mexican members of the family Gleicheniaceae was carried out. The material was collected in several locations of the country, morphological descriptions were made with optic and scanning electron microscopes, and this work includes images of all the studied species. Results show differences between species in laesure type and surface ornamentation. The micromorphology of the spores of S. brevipubis, S. palmatus, and $S$. underwoodianus are described for the first time.

Key words: ornamentation, Pteridophyta, spores, scanning electron microscope.
${ }^{1}$ Departamento de Biología, Área de Botánica Estructural y Sistemática Vegetal, Universidad Autónoma Metropolitana, Unidad Iztapalapa, México, D. F., México

${ }^{2}$ Facultad de Ciencias, Laboratorio de Plantas Vasculares, Universidad Nacional Autónoma de México, México, D. F., México

${ }^{3}$ Autor para la correspondencia:amr@xanum.uam.mx 
a palinología es una herramienta importante en el estudio de la flora, ya que, entre otros casos, aporta información útil en estudios taxonómicos. Por ejemplo, la morfología y micromorfología de las esporas de los helechos aportan caracteres como el tamaño, la forma y la ornamentación del perisporio, los cuales pueden ayudar a resolver problemas de delimitación taxonómica a nivel de familia, género y en algunos casos especie (Lellinger y Taylor, 1997). Adicionalmente, la información que los palinomorfos nos brindan puede ser empleada en estudios de tipo ecológico, fenológico y dispersión, como indicadores geológicos y paleoecológicos del registro fósil y en el campo forense (Harris, 1957; Vaughn et al., 1990).

Las Gleicheniaceae, por sus características anatómicas, morfológicas y por el registro fósil, se consideran dentro del grupo de los helechos leptosporangiados basales, apareciendo por primera vez durante el Cretácico, entre 144 y 65 millones de años atrás (Wallace et al., 1983; Thomas y Spicer, 1987; Gandolfo et al., 1997; Herendeen y Skog, 1998; Moran, 2004); comprende plantas cuyos rizomas son postrados con estela de tipo protostélico o solenostélico; láminas divididas pseudodicotómicamente (excepto en Stromatopteris Mett.); venación libre bifurcada; esporangios globoso con un anillo oblicuo y esporas tetraédricas o bilaterales (Smith et al., 2006).

De las 125 especies reconocidas actualmente en la familia (Smith et al., 2006), sólo Dicranopteris flexuosa (Schrad.) Underw., Diplopterygium bancroftii (Hook.) A. R. Sm., Gleichenella pectinata (Willd.) Ching, Sticherus bifidus (Willd.) Ching, S. brevipubis (Christ) A. R. Sm., S. palmatus (W. Schaffn. ex E. Fourn.) Copel. y S. underwoodianus (Maxon) Nakai, se encuentran en México (Smith et al., 2006).

La circunscripción de la familia Gleicheniaceae ha sido controvertida. Así por ejemplo, Christensen (1906) reconoce dos géneros: Gleichenia Sm. y Stromatopteris y divide a este último en tres subgéneros, posteriormente Underwood (1907) acepta además de los dos taxa anteriores a Dicranopteris Bernh. y Platyzoma R.Br., por su parte Christensen (1938) reconoce los cuatro géneros previos y adiciona a Sticherus C. Presl., Ching (1940) y Copeland (1947) siguen la propuesta de Christensen (1938), incluyendo además el primer autor a Gleichenella Ching y el segundo a Hicriopteris C.Presl., Nakai (1950) segrega a Platyzoma en su propia familia, Platyzomaceae, y Gleicheniaceae queda integrada por tres subfamilias y nueve géneros. Holttum (1957) divide al grupo en dos géneros: Dicranopteris y Gleichenia con dos y tres subgéneros respectivamente. Sin embargo, en las clasificaciones más recientes, Smith et al. (2006) y Christenhusz et al. (2011) reconocen para las Gleicheniaceae seis géneros a nivel mundial, Dicranopteris, Diplopterygium, Gleichenella, Gleichenia, Sticherus y Stromatopteris. La inclusión de este último género se ve apoyada por los estudios fitoquímicos realizados por Wallace et al. (1983) y Yusuf et al. (2003), en los que se demuestra la presencia de compuestos fenólicos (kaempferol y quercetina) en 16 especies de Gleicheniaceae incluyendo a Stromatopteris.

En lo que se refiere al estudio palinológico, son diversos los trabajos que incluyen información sobre la morfología de las esporas de alguna de las especies de la familia Gleicheniaceae (Erdtman y Sorsa, 1971; Lugardon, 1974; Tryon y Tryon, 1982; Kramer, 1990; Tryon y Lugardon, 1991; Palacios-Ríos, 1992; Velázquez-Montes, 2012), sin embargo los estudios realizados no abarcan la totalidad de las especies mexicanas. Es por esta razón que en el presente trabajo, utilizando microscopía electrónica de barrido (MEB), se describe la micromorfología de las esporas de las especies de Gleicheniaceae que crecen en México, describiéndose por primera vez las esporas de Sticherus brevipubis, S. palmatus y S. underwoodianus con base a fotomicrografías tomadas bajo MEB.

\section{Materiales y métodos}

Los ejemplares de respaldo y pinnas fértiles, fueron recolectados en distintas localidades de los estados de Oaxaca, Puebla y Veracruz (Tabla 1). Los especímenes se determinaron taxonómicamente con base en Mickel y Smith (2004) y se cotejaron con material de diferentes herbarios, entre ellos MEXU y UAMIZ, para corroborar su correcta determinación, una vez procesados se depositaron en la colección de los herbarios arriba mencionados. Las pinnas fértiles fueron colocadas en sobres de papel manila, los cuales fueron rotulados y colocados en estantes de tipo esqueleto durante una semana para propiciar la liberación de las esporas. Posteriormente se hizo 
Tabla 1. Especies de la familia Gleicheniaceae recolectadas en varias localidades de México (BE = Bosque de encino, BPE = Bosque pino-encino, $\mathrm{BMM}=$ Bosque mesófilo de montaña, BTC = Bosque tropical caducifolio, con base en Rzedowski, 2006).

\begin{tabular}{ll} 
Especies & $\begin{array}{l}\text { Colector y No. } \\
\text { de colecta }\end{array}$ \\
\hline Dicranopteris flexuosa & N. Farfán S. 30 \\
& N. Farfán S. 31 \\
Diplopterygium bancroftii & N. Farfán S. 1 \\
N. Farfán S. 6 \\
N. Farfán S. 16 \\
Gleichenella pectinata & N. Farfán S. 7 \\
& N. Farfán S. 21 \\
& N. Farfán S. 29 \\
Sticherus bifidus & N. Farfán S. 8 \\
& N. Farfán S. 9 \\
& N. Farfán S. 22 \\
N. Farfán S. 25
\end{tabular}

Sticherus brevipubis

N. Farfán S. 17

N. Farfán S. 23

Sticherus palmatus

N. Farfán S. 2

N. Farfán S. 10

N. Farfán S. 15

N. Farfán S. 20

Sticherus underwoodianus N. Farfán S. 3

N. Farfán S. 4

N. Farfán S. 5
Localidad

- 9 km después de Putla, rumbo a Pinotepa Nacional, Mpio. Putla de Villa de Guerrero, Oaxaca.

- 7 km después de San Marcos Coyulito, 2.8 km antes de llegar a La Muralla, carretera Pinotepa Nacional- Putla de Guerrero, Mpio. Putla de Villa de Guerrero, Oaxaca.

- Desviación a Yaonáhuac, ca. de 8 km a partir del entronque de la carretera Tlatlauquitepec-Mazatepec, Mpio.

Tlatlauquitepec, Puebla.

- 4 km después de La Legua, carretera Teziutlán-Tlapacoyan, Mpio. Teziutlán, Puebla.

- Puente el Tablazo, entrada a rancho Maroyut, 9 km después de Atzalán sobre la carretera Altotonga-Tlapacoyan, Mpio. Atzalán, Veracruz.

- 1 km después de Cruz Verde, hacia Hueytamalco, Mpio. Hueytamalco, Puebla.

- Rancho San Bartolo, carretera estatal Tlaltetela-Totutla, 7 km después de Ohuapan, Mpio. Tlaltetela, Veracruz.

- 2.5 km después de Llano San Vicente, rumbo a Putla, Mpio. Putla Villa de Guerrero, Oaxaca.

Tipo de vegetación y altitud (m s.n.m.)

BPE, 752

BTC, 963

BPE, 1,653

BMM, 1,524

BMM, 1,140

BMM, 1,113

BMM, 1,438

BPE, 1,005

BMM, 1,627

- Lalancingo, 6.8 km después de Arrollo Tendido, rumbo a Tlapacoyan, Mpio. Hueytamalco, Puebla.

- $3 \mathrm{~km}$ antes de llegar a Cuetzalan, viniendo de Ayotoxco de Guerrero, Mpio. Cuetzalan, Puebla.

- $3 \mathrm{~km}$ antes de llegar a Huatusco sobre la carretera

Totutla-Huatusco, Mpio. Huatusco, Veracruz.

- 7.5 km después de San Juan Coatzóspam sobre la carretera federal Huahutla-San Felipe Jalapa de Díaz, Mpio. San Juan Coatzóspam, Oaxaca.

BMM, 980

BMM, 1310

BE, 1,390

BMM, 1,140

- Puente el Tablazo, entrada a rancho Maroyut, 9 km después de Atzalán sobre la carretera Altotonga-Tlapacoyan, Mpio. Atzalán, Veracruz.

- 1.2 km después de Plan de Guadalupe, rumbo a Huautla de Jiménez, sobre la carretera Teotitlán-Huautla, Mpio., Santa María Teopoxco, Oaxaca.

$\mathrm{BE}, 1,239$

BMM, 1,627

- Entronque a San José Chagchalzin, carretera TlatlauqiutepecMazatepec, Mpio. Tlatlauquitepec, Puebla.

- Camino entre Cascada las Brisas y Cascada Velo de Novia, Mpio. Cuetzalan, Puebla.

- Puente el Tablazo, entrada a rancho Maroyut, 9 km después de Atzalán sobre la carretera Altotonga-Tlapacoyan en dirección a Ocotepec, Mpio. Atzalán, Veracruz.

- 8 km después de Pinillo, sobre la carretera estatal Tlaltetela-

Totutla, Mpio. Tlaltetela, Veracruz.

BMM, 928

BMM, 1,140

$\mathrm{BE}, 1,439$

- Vereda que sale de Xucayucan, rumbo a la cascada de Puxtla, carretera Tlatlauquitepec-Mazatepec, Mpio. Tlatlauquitepec, Puebla. - La Legua, km 5 de la carretera Teziutlán- Tlapacoyan, Mpio. Teziutlán, Puebla.

- 4 km después de La Legua, carretera Teziutlán-Tlapacoyan, Mpio. Teziutlán, Puebla.
BMM, 1,627

BMM, 1,678

BMM, 1,524

pasar el contenido de los sobres por un tamiz con una malla de $74 \mu \mathrm{m}$ de diámetro para eliminar restos de esporangios, pelos y/o escamas. Las esporas se depositaron en viales de plástico y se almacenaron bajo condiciones de refrigeración a $10{ }^{\circ} \mathrm{C}$ para conservarlas. Para las observaciones en el microscopio electrónico de barrido, se utilizaron pinceles de pocas cerdas con los que se espolvoreó una pequeña cantidad de esporas de cada especie sobre cinta de carbón de doble cara adherida a portamuestras de aluminio. Estas se cubrieron con una capa de oro-paladio 


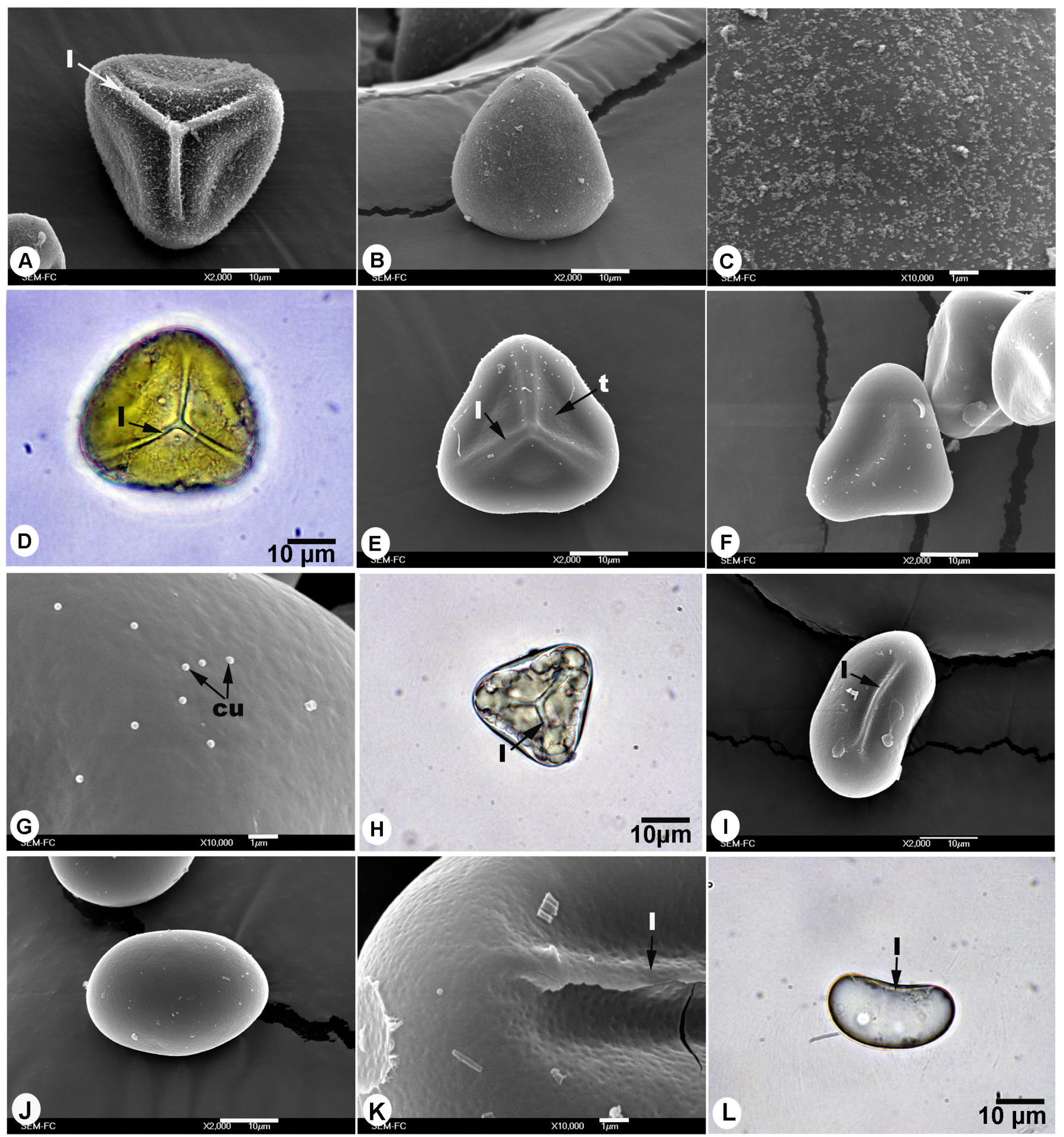

Figura 1. Esporas de Gleicheniaceae. A-D) Espora trilete de Dicranopteris flexuosa, A) Vista proximal, B) Vista distal, C) Acercamiento de superficie, D) Vista proximal en microscopía óptica. E-H) Espora trilete de Diplopterygium bancroftii, E) Vista proximal, F) Vista distal, G) Acercamiento de la superficie, F) vista proximal, H) vista proximal en microscopía óptica. I-L) Espora monolete de Gleichenella pectinata, I) Vista proximal, J) Vista distal. K) Acercamiento de la superficie. L) Vista ecuatorial en microscopía óptica. cu = cuerpos de Ubish, $\mathrm{l}=$ lesura, $\mathrm{t}=$ torus.

utilizando una ionizadora Denton Vacuum Desk II. Una vez procesadas las muestras, fueron llevadas al Microscopio Electrónico de Barrido JEOL JSM-5310LV a 15 kv, donde se tomaron fotomicrografías de las esporas en vista proximal, distal, así como el acercamiento de la super- 

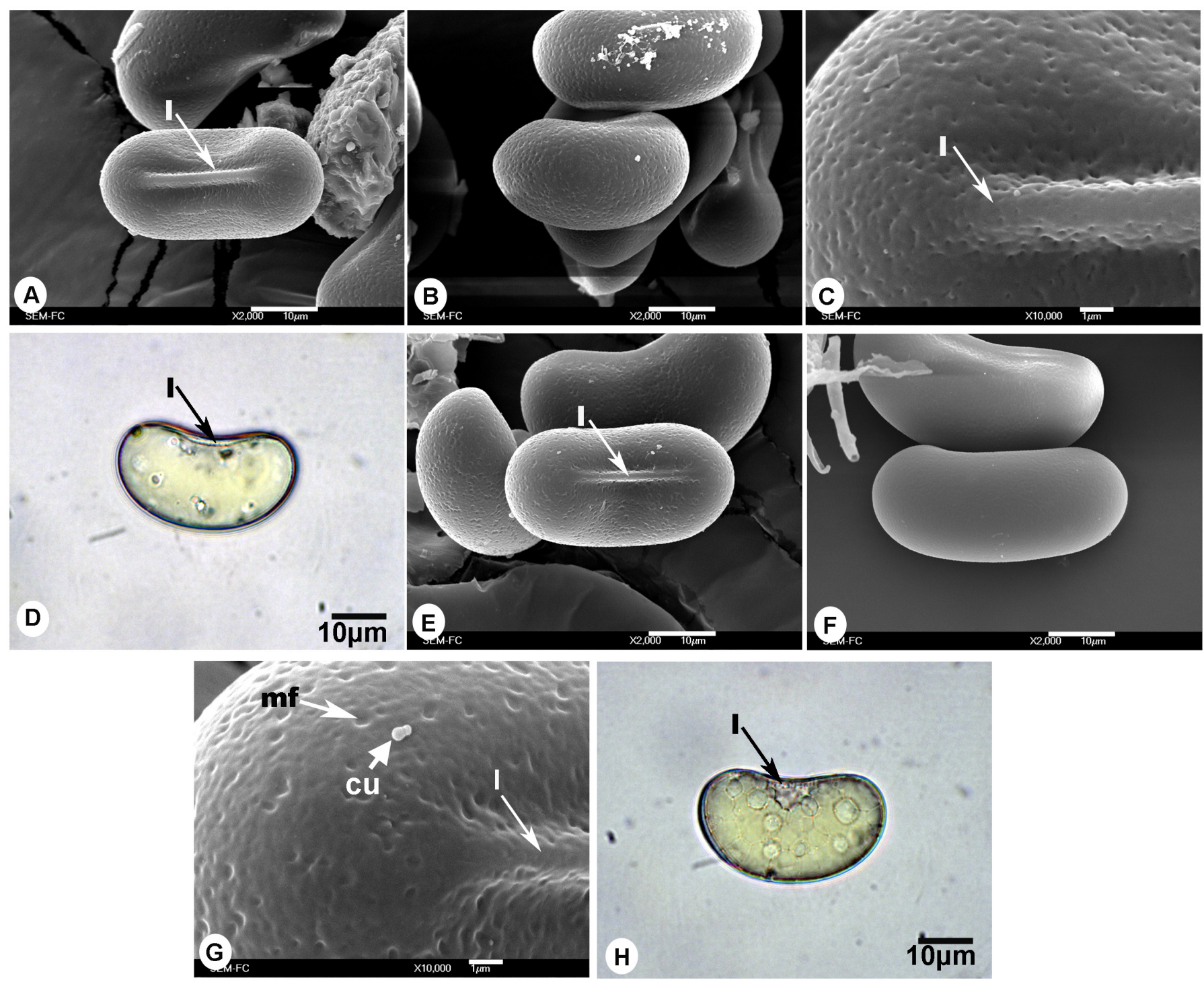

Figura. 2. Esporas de Gleicheniaceae. A-D. Espora monolete de Sticherus bifidus, A) Vista proximal, B) Vista distal, C) Acercamiento de la superficie, D) Vista ecuatorial en microscopía óptica. E-H) Espora monolete de Sticherus brevipubis, E) Vista proximal, F) Vista distal,

G) Acercamiento de la superficie, H) Vista ecuatorial en microscopía óptica. cu = cuerpos de Ubish, $1=$ lesura, $\mathrm{mf}=$ microfoveolas.

ficie de las esporas. Las imágenes digitales se obtuvieron con el sistema Orion-Jeol y fueron procesadas con el programa Adobe Photoshop CS5.

Adicionalmente se obtuvieron imágenes con un microscopio óptico Olympus modelo BX41 (MO), así mismo se seleccionaron al azar 25 esporas por especie, de las que se registró la coloración y se tomaron medidas, en el caso de las monoletes, de los ejes polar y ecuatorial y de las triletes sólo del eje ecuatorial, obteniéndose en cada caso el tamaño promedio.

\section{Resultados}

Todas las especies estudiadas son homospóricas, dos de ellas presentan esporas triletes y las cinco restantes monoletes. A continuación se describe la micromorfología, ornamentación y tamaño (mínimo, promedio, máximo y \pm desviación estándar) de cada uno de los taxa de la familia Gleicheniaceae que crecen en México, ilustrando cada una de las especies con imágenes de las esporas obtenidas con el microscopio electrónico de Barrido (MEB) y con microscopio óptico (MO). 

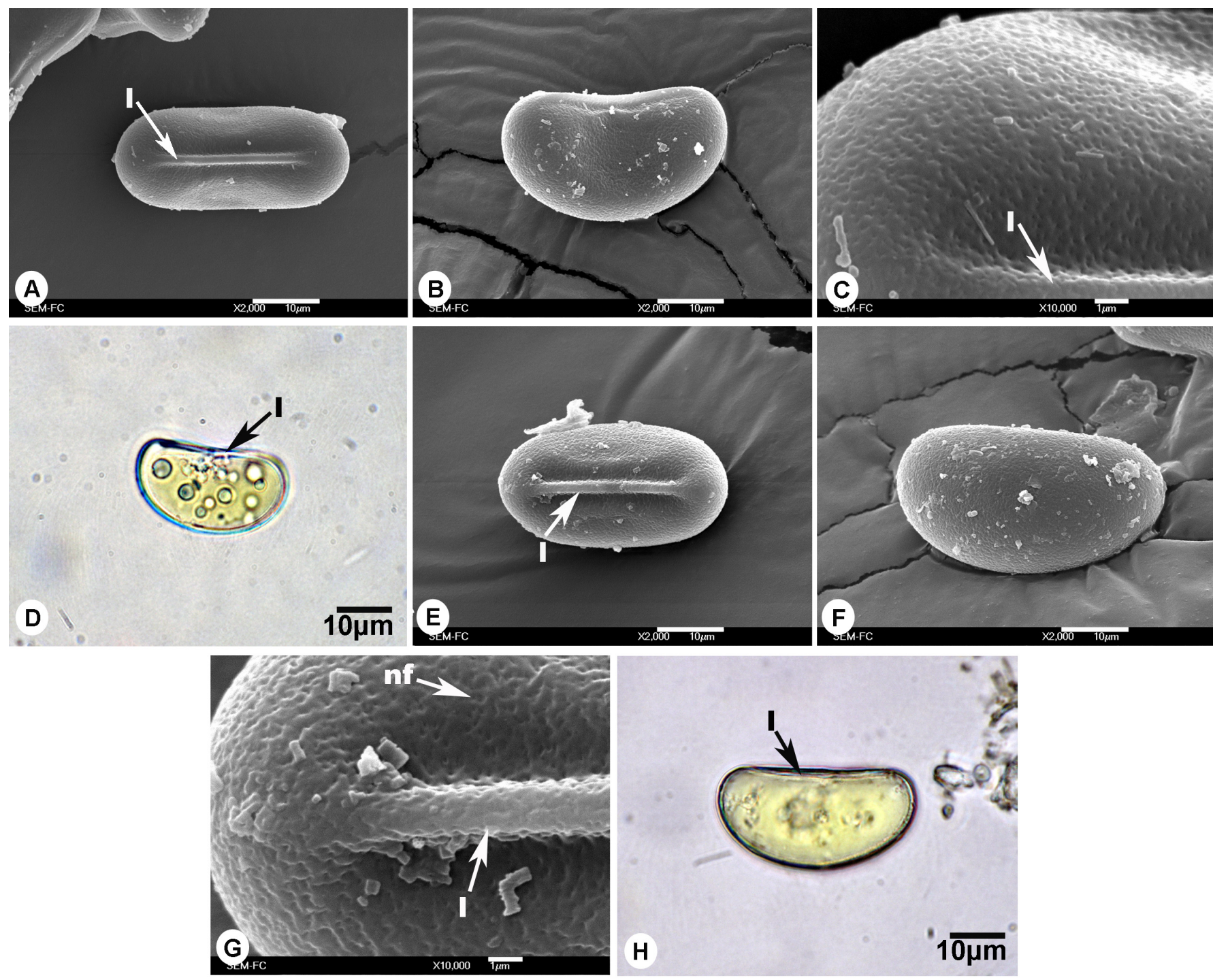

Figura 3. Esporas de Gleicheniaceae. A-D. Espora monolete de Sticherus palmatus, A) Vista proximal, B) Vista ecuatorial, C) Acercamiento de la superficie, D) Vista ecuatorial en microscopía óptica. E-H) Espora monolete de Sticherus underwoodianus, E) Vista proximal, F) Vista distal, G) Acercamiento de la superficie, H) Vista ecuatorial en microscopía óptica. 1 = lesura, nf = nanofoveolas.

Dicranopteris flexuosa (Schrad.) Underw.

Esporas triletes; vista polar: forma subtriangular, contorno convexo-triangular, lados convexos, ángulos redondeados, lesura prominente $>3 / 4$ del radio, torus presente; ornamentación isopolar: caras proximal y distal escábridas, elementos irregulares $<1 \mu \mathrm{m}$ (Figura 1A-C); eje ecuatorial de 25 (32) $38 \pm 3.7 \mu \mathrm{m}$; coloración amarillenta a simple vista y al MO (Figura 1D).

Diplopterygium bancroftii (Hook.) A. R. Sm.

Esporas triletes; vista polar: forma triangular, contorno triangular ligeramente cóncavo, lados rectos a ligeramente cóncavos, ángulos redondeados, lesura prominente $>3 / 4$ del radio, zonas de contacto evidentemente deprimidas, formando lagunas triangulares; ornamentación isopolar: caras proximal y distal psiladas con escasos gránulos orbiculares denominados cuerpos de Ubish (MEB, Figura 1E-G); eje ecuatorial de 25 (31) $37 \pm 3.2 \mu \mathrm{m}$; coloración amarillo pálido a simple vista, hialinas en MO (Figura $1 \mathrm{H}$ ).

Gleichenella pectinata (Willd.) Ching

Esporas monoletes; vista polar: forma elipsoidal; vista ecuatorial: forma reniforme, polo proxi- 
mal plano a cóncavo, polo distal convexo; lesura lineal prominente de menos de $1 \mu \mathrm{m}$ de ancho y extendida $1 / 2$ del eje ecuatorial; ornamentación isopolar: caras proximal y distal psilada (MO), nanofoveolada (MEB), foveolos $<0.5 \mu \mathrm{m}$ con escasos cuerpos de Ubish (MEB, Figura 1I-K); eje ecuatorial de 28 (32) $38 \pm 2.7 \mu \mathrm{m}$, eje polar de 13 (18) $23 \pm 2.2 \mu \mathrm{m}$; coloración blanquecina a simple vista, hialinas al MO (Figura 1L).

Sticherus bifidus (Willd.) Ching

Esporas monoletes; vista polar: forma elipsoidal; vista ecuatorial: reniforme, polo proximal cóncavo, polo distal convexo; lesura lineal prominente de 1.5 a $2 \mu \mathrm{m}$ de ancho, mayor a $1 / 2$ el eje ecuatorial; ornamentación isopolar: caras proximal y distal nanofoveolada a perforada (MEB, Figura 2A-C) con escasos cuerpos de Ubish; eje ecuatorial de 33 (39) $45 \pm 3.2 \mu \mathrm{m}$, eje polar de 15 (19) $25 \pm 2.6 \mu \mathrm{m}$; coloración amarilla a simple vista, hialina a amarillo pálido en MO (Figura 2D).

Sticherus brevipubis (Christ.) A. R. Sm.

Esporas monoletes; vista polar: elipsoidal; vista ecuatorial: reniforme, polo proximal cóncavo, polo distal convexo; lesura lineal prominente, de 1 a $1.5 \mu \mathrm{m}$, extendida de $1 / 3$ a $1 / 2$ del eje ecuatorial; ornamentación isopolar: caras proximal y distal microfoveolada a perforada (MEB, Figura 2F-G) con escasos cuerpos de Ubish; eje ecuatorial de 30 (38) $48 \pm 4.9 \mu \mathrm{m}$, eje polar de 15 (19) $25 \pm 2.6 \mu \mathrm{m}$; coloración pardo claro-amarillenta a simple vista, hialinas MO (Figura $2 \mathrm{H}$ ).

Sticherus palmatus (W. Schaffn. ex E. Fourn.) Copel.

Esporas monoletes; vista polar: forma elipsoidal, lados rectos; vista ecuatorial: reniforme, polo proximal plano a ligeramente cóncavo, polo distal convexo; lesura lineal prominente $>3 / 4$ del eje ecuatorial; ornamentación isopolar: caras proximal y distal nanofoveolado (MEB, Figuras 3A-C); eje ecuatorial de 30 (36) $43 \pm 3.3 \mu \mathrm{m}$, eje polar de 13 (17) $20 \pm 1.8 \mu \mathrm{m}$; coloración amarilla a simple vista, amarillo-pálido MO (Figura 3D).

\section{Sticherus underwoodianus (Maxon) Nakai}

Esporas monoletes; vista polar: forma elipsoidal, lados planos a convexos; vista ecuatorial: reniforme, polo proximal recto, polo distal convexo; lesura lineal prominente de 1.5 a $2 \mu \mathrm{m}$ de ancho, mayor a $3 / 4$ el eje ecuatorial; ornamentación isopolar: caras distal y proximal hamulada (rugulas irregulares) con nanofoveolos (MEB, Figura 3E-G); eje ecuatorial de 33 (40) $45 \pm$ $3.0 \mu \mathrm{m}$, eje polar de 15 (20) $28 \pm 2.9 \mu \mathrm{m}$; coloración blanquecina a amarillenta a simple vista, amarilla a hialina en $\mathrm{MO}$ (Figura $3 \mathrm{H}$ ).

\section{Discusión}

Las observaciones de este trabajo coinciden con lo señalado en otros sobre la apertura de las esporas de especies de la familia (Erdtman y Sorsa, 1971; Lugardon, 1974; Tryon y Tryon, 1982; Kramer, 1990; Tryon y Lugardon, 1991; Palacios-Ríos, 1992; Velázquez-Montes, 2012), en el entendido de que las esporas en Dicranopteris flexuosa y Diplopterygium bancroftii son triletes; mientras que en Gleichenella pectinata, Sticherus bifidus, S. brevipubis, S. palmatus y S. underwoodianus son monoletes.

En cuanto al tamaño de las esporas triletes, las de Dicranopteris flexuosa y Diplopterygium bancroftii, presentan dimensiones similares con 32-31 $\mu \mathrm{m}$ de diámetro respectivamente; mientras que las esporas monoletes de Gleichenella pectinata fueron las más pequeñas con $32 \mu \mathrm{m}$ de largo (vista ecuatorial) $\times 18 \mu \mathrm{m}$ de ancho (vista polar) y las de Sticherus las más grandes con 36-49 $\mu \mathrm{m}$ en vista ecuatorial $\times 17-20 \mu \mathrm{m}$ en vista polar.

En lo que se refiere a la ornamentación de las esporas, se encontró que las de Diplopterygium bancroftii, Gleichenella pectinata y las especies de Sticherus presentan cuerpos de Ubish en su superficie, mientras que las de Dicranopteris flexuosa son microgranuladas o escábridas (Tryon y Tryon, 1982; Kramer, 1990; Tryon y Lugardon, 1991) y no psiladas a ligeramente ruguladas como lo indica Velázquez-Montes (2012), lo anterior pueden deberse a las técnicas empleadas para examinar el material, ya que en el último trabajo citado las descripciones se realizaron 
con base a observaciones hechas con microscopio óptico, mientras que en los demás se utilizó microscopio electrónico de barrido.

Las esporas de Diplopterygium bancroftii presentan una superficie predominantemente psilada con cuerpos de Ubish dispersos lo cual concuerda con trabajos previos para esta especie (Lugardon, 1974; Tryon y Lugardon, 1991; Velázquez-Montes, 2012). Por su parte las de Gleichenella pectinata poseen una superficie predominantemente psilada a ligeramente nanofoveolada con escaso cuerpos de Ubish. La presencia de nanofoveolos y cuerpos de Ubish no habían sido reportada para esta especie en estudios previos (Erdtman y Sorsa, 1971; Tryon y Lugardon, 1991); en el caso de las esporas de Sticherus se observó que las de S. bifidus y S. palmatus tienen una superficie perforada o nanofoveolada coincidiendo con los reportes de Tryon y Tryon (1982), Kramer (1990) y Tryon y Lugardon (1991) y no lisas como las describen Erdman y Sorsa (1971) para S. bifidus, mientras que las de S. brevipubis son perforadas y las de S. underwoodianus son perforadas o ruguladas.

Tomando en cuenta los resultados obtenidos en este trabajo, se considera que las Gleicheniaceae mexicanas pueden separarse a nivel genérico y en algunos casos a nivel específico, por la forma monolete o trilete, tipo de lesura, ornamentación del exosporio o perisporio y por el tamaño de sus esporas.

Cabe señalar que la micromorfología de las esporas de Sticherus brevipubis, S. palmatus y $S$. underwoodianus se describe por primera vez aquí, presentando imágenes de Microscopio Electrónico de Barrido que aportan información nueva y detallada respecto a la ornamentación de las esporas de Gleicheniaceae.

Las descripciones detalladas de la micromorfología de las esporas son útiles en los tratamientos taxonómicos de los helechos, ya que ayudan a distinguir géneros y en algunos casos especies como en el caso de las Gleicheniaceae mexicanas y para caracterizar a las familias. El tamaño y forma irregular también permiten distinguir grupos poliploides e híbridos (Brown, 1960). Finalmente el conocer con mayor detalle la micromorfología de las esporas de los helechos se aportan datos para realizar estudios de dispersión y lluvia de esporas (Gómez-Noguez et al., 2013) ya que nos ayuda a identificar las esporas recolectadas en el campo de manera más precisa.

\section{Agradecimientos}

Se agradece a Jacqueline Ceja Romero, Felipe Gómez Noguez y a los revisores anónimos sus comentarios al trabajo, a Silvia Espinosa Matías del laboratorio de Microscopía Electrónica de la Facultad de Ciencias, UNAM, por el apoyo en el procesamiento y obtención de las imágenes en MEB. Al CONACYT por el apoyo con la beca de posgrado (No. Becario 265990) del primer autor, a la maestría en Biología de la Universidad Autónoma Metropolitana Unidad Iztapalapa y al proyecto Fondo SEP-CONACYT 105536.

\section{Literatura citada}

Brown C.A. 1960. What is the role of spores in fern taxonomy? American Fern Journal 50:6-14.

Ching R.C. 1940. On the genus Gleichenia Smith. Sunyatsenia 5:269-288.[RV2]

Christenhusz M.J.M., Zhang X.-C. y Schneider H. 2011. A linear sequence of extant families of lycophytes and ferns. Phytotaxa 19:7-54.

Christensen C. 1906. Index Filicum, sive enumeratio omnium generum specierumque Filicum et Hydropteridum ab anno 1753 ad finem anni 1905 descriptorum adjentis synonymis principalibus, area geographica etc. H. Hagerup, Copenhage..

Christensen C. 1938: Filicinae. En: Verdoorn F. y Alston A.H.G. Eds. Manual of Pteridology, pp. 522-550, Nijhoff., The Hague.

Copeland E.B. 1947. Genera Filicum: the genera of ferns. En: Verdooorn F. Ed. Annales Cryptogamici et Phytopathologici, pp. 26-28, Chronica Botanica Company of Waltham, Massachusetts. [RV3]

Erdtman G. y Sorsa P. 1971. Pollen and Spore Morphology/Plant Taxonomy: Pteridophyta. Almqvist \& Wiksell, Estocolmo.

Gandolfo M.A., Nixon K.C., Crepet W.L. y Ratcliffe G.E. 1997. A new fossil fern assignable to the Gleicheniaceae from late cretaceous sediments of New Jersey. American Journal of Botany 84:483-493.

Gómez-Noguez F., Pérez-García B., Mendoza-Ruiz A. y Orozco-Segovia A. 2013. Flora palinológica de los helechos y licofitas de Río Malila, Hidalgo, México. Botanical Sciences 91:135-154. 
Recibido:

26 de diciembre de 2014

Aceptado:

3 de marzo de 2015
Harris W.F. 1957. A manual of the spores of New Zealand Pteridophyta. American Fern Journal 49:114116.

Herendeen P.S. y Skog E.J. 1998. Gleichenia chaloneri- A New Fossil Fern from the Lower Cretaceous (Albian) of England. International Journal of Plant Sciences 159:870-879.

Holttum R.E. 1957. Florae Malesianae Praecursores XVI. On the taxonomic subdivision of the Gleicheniaceae, with descriptions of new Malasyan species and varieties. Reinwardtia 4:257-280.

Kramer K.U. 1990. Gleicheniaceae. En: Kubitzki K., Kramer K.U. y Green P.S. Ed. The Families and Genera of Vascular Plants. Volumen I, Pteridophytes and Gymnosperms, pp. 145-152. Springer-Verlag, Berlin-Heidelberg-Nueva York

Lellinger D.B. y Taylor W.A. 1997. Classification of spore ornamentation in the Pteridophyta. En: Johns R.J. Ed. Holttum Memorial Volume: published to commemorate the centenary of the birth of Professor R. E. Holttum, pp. 32-43, Royal Botanical Gardens, Kew.[RV6]

Lugardon B. 1974. La structure fine de l'exospore et de la perispore des Filicinées isosporées. Pollen \& Spores 16:161-226.

Mickel J.T. y Smith A.R. 2004. The pteridophytes of Mexico. Memoirs of the New York Botanical Garden 88:1-1054.

Moran R.C. 2004. Fern fossils. En: Moran R.C. A Natural History of Ferns, pp. 13-129, Timber Press, Portland.

Nakai T. 1950. A new classification of Gleicheniaceae. Bulletin of the National Science Museum Tokyo 29:1-71.

Palacios-Ríos M. 1992. Gleicheniaceae. Flora de Veracruz, Fascículo 69:23-45.

Rzedowski J. 2006. Vegetación de México.. Comisión Nacional para el Conocimiento y Uso de la Biodiversidad, México D.F.

Smith A.R., Pryer K.M., Schuettpelz E., Korall P., Schneider H. y Wolf P.G. 2006. A Classification for Extant Ferns. Taxon 55:705-731.

Thomas B.A. y Spicer R.A. 1987. The Evolution and Paleobiology of Land Plants Vol. 2, pp. 121-138, Dioscorides Press, Portland.

Tryon A.F. y Lugardon B. 1991. Spores of the Pteridophyta. Surface, Wall Structure and Diversity Based on Electron Microscope Studies. Springer-Verlag, Nueva York.

Tryon M.R. y Tryon A.F. 1982. Ferns and Allied Plants with special reference to Tropical America. Springer-Verlag, Nueva York.

Underwood L.M. 1907. American ferns VIII. A preliminary review of the North American Gleicheniaceae. Bulletin of the Torrey Botanical Club 34:243-262.

Vaughn M.B. Jr., Jones J.G. y Mildenhall D.C. 1990. Forensic Palynology in the United States of America. Palynology 14:193-208.

Velázquez-Montes E. 2012. Gleicheniaceae. En: ¿? Flora de Guerrero No. 53, Prensas de Ciencias, Universidad Nacional Autónoma de México, México D.F.

Wallace J.W., Pozner R.S. y Gómez L.D. 1983. A phytochemical approach to the Gleicheniaceae. American Journal of Botany 70:207-211.

Yusuf U., Itam K., Abdullah F., Zainal I. y Sukari M.A. S. 2003. Leaf Flavonoids on the Genus Gleichenia (Gleicheniaceae). American Fern Journal 93:44-46. 